We wish to acknowledge financial assistance from the Rural Credits Division of the Commonwealth Bank of Australia. The work was carried out while one of us (J. S.) held the I.C.I.A.N.Z. Research Fellowship of the University of Tasmania.

JANET I. SPRENT

University of Tasmania,

H. N. Barber

Hobart. March 25.

1 Barber, H. N., and Paton, D. M., Nature, 169, 592 (1952).

2 Paton, D. M., and Barber, H. N., Austral. J. Biol. Sci., 8, 231 (1955)

\section{Partial Enzymic Degradation of Tamarindus-Amyloid}

HeateD cellulase of Myrothecium verrucaria ${ }^{1}$ degrades the amyloid of Tamarindus indica to a product which by means of paper chromatography (applying butanol/pyridine/water/benzene (5:3.2: $3: 0.45)$ as a solvent and developing for a week) appears to contain at least eight oligosaccharides, three of them occurring as the main products. These three oligosaccharides, $A, B$ and $C$, could be shown to be a hepta., an octa- and a nona-saccharide respectively, and are composed of 4 glucose residues, 3 xylose residues and 0,1 and 2 galactose residues respectively. They are liberated in a molar ratio of roughly $2: 5: 6$. The three oligosaccharides constitute a homologous series; this was confirmed by the facts that: (1) the values of $\log R_{F} /\left(1-R_{F}\right)$ lie on a straight line when plotted against degree of polymerization $^{1}$, and (2) the values of $[M] n / n$, when plotted against $(n-1) / n$, lie on a straight line as well ${ }^{2}$.

Amyloids from seeds of other plant species can also be hydrolysed by cellulase. On paper chromatogxams the oligosaccharides from the amyloids investigated (from Tropaeolum majus, Cyclamen hybr., Impatiens balsamina, Hymenaea courbaril, Schotia latifolia) have mobilities which suggest that in all instances the oligosaccharides are the same as those from the amyloid of Tamarindus. The ratios in which the oligosaccharides appear are different for different species. Tropaeolum amyloid is hydrolysed by cellulase to give the three oligosaccharides in a molar ratio of roughly $1: 2: 10$. Cyclamen amyloid gives the oligosaccharides $A, B, C$, and $D$ in a molar ratio of approximately $2: 7: 2: 2$. (Oligosaccharide $D$ occurs in cellulase hydrolysates of Tamarindus and Tropaeolum amyloids only in negligible amounts, whereas in Cyclamen amyloid the amount is much larger.)

The specific rotations of the oligosaccharides $C$ from the amyloids of Tamarindus, Tropaeolum and Cyclamen are: $[\alpha]_{D}^{188^{\circ} \mathrm{C}}=73 \cdot 0^{\circ}, 74 \cdot 6^{\circ}$ and $75 \cdot 6^{\circ}$ respectively $(c=1$, water $)$. The correspondence of the specific rotations and of the chromatographic behaviour of the oligosaccharides $C$ suggests that they are identical. Oligosaccharides $A$ may also be identical, as well as the oligosaccharides $B$.

It is apparent that the amyloids of seeds have constitutions which are highly interrelated.

Details of this work will be published elsewhere. P. KOOIMAN

Laboratory for General and Technical Biology, Technical University, Delft. March 21.

1 Kooiman, P., and Roelofsen, P. A., Enzymologia, 16, 237 (1953).

${ }^{2}$ French, D., and Wild, G. M., J. Amer. Chem. Soc., 75, 2612 (1953).

"French, D., "Adv. Carbohydrate Chem.", 9, 160 (1954).

\section{Formation of $\beta-1,4-$ Oligoglucosides by Certain Acetobacter Species}

RECENT studies ${ }^{1}$ in these laboratories have revealed the presence of cellobiose, cellotriose and cellotetraose in cellulose-producing cultures of Acetobacter acetigenum, NCIB 8132. The present communication deals with an extension of this work in which other cellulose-producing cultures were examined for the presence of $\beta$-1,4-oligoglucosides. Mutant organisms unable to produce extracellular cellulose (celluloseless mutants) were also studied.

The cellulose-producing cultures examined were Acetobacter acetigenum, NCIB 8132, A. xylinum, NCIB 8034 and $A$. xylinum var. africanum, NCIB 7029 ; these cultures were obtained from the National Collection of Industrial Bacteria, Teddington, Middlesex. Cultures of celluloseless mutants were isolated from the wild-type cultures by methods described previously ${ }^{2}$; these are 'natural' mutants which occur spontaneously, that is, without treatment of the parent culture with mutagenic agents. Two mutant cultures were examined from each of organisms NCIB 8132 and NCIB 8034 and one from organism $N C I B$ 7029. The parent and the mutant organisms were grown in a medium $(p H 6.0)$ which contained the following components (per cent, $w / v$ ) in distilled water : glucose, 3.0 ; dibasic potassium phosphate, 0.3 ; ammonium sulphate, 0.3 ; magnesium sulphate heptahydrate, 0.2 ; calcium pantothenate, 0.0002 ; riboflavin, 0.0002 ; and biotin, $0 \cdot 00003$. One litre of medium, dispensed at $250 \mathrm{ml} . /$ mould cultureflask $k^{3}$, was inoculated with the desired test organism and after ten days incubation at $28^{\circ}$ the contents of the four flasks were pooled and processed as follows. The bacteria were removed by Seitz filtration of the medium, solid barium carbonate $(0.5 \mathrm{gm}$.) was added, the solution was concentrated (in racuo, below $40^{\circ}$ ) to about $200 \mathrm{ml}$. and then passed through a charcoal'Celite' column ${ }^{4}$ to separate the oligosaccharides. Elution with water, $7 \cdot 5$ per cent $(\mathrm{v} / \mathrm{v})$ ethanol and 20 per cent ethanol (1.5 I. of each), respectively, gave fractions 1-3. A fourth fraction was obtained by boiling the charcoal-'Celite' mixture in 30 per cent ethanol ${ }^{5}$. All fractions were concentrated to about $20 \mathrm{ml}$. (in vacuo, below $40^{\circ}$ ), deionized with 'Zeo-Karb $225(\mathrm{H})$ ' and then with 'Amberlite $I R-4 B$ (OH)'. After removal of the resin, the solutions were concentrated to syrups and examined for the presence of oligosaccharides by paper chromatography ${ }^{8}$.

A sugar component with an $R_{F}$ value similar to that of authentic glucose was found in all fractions, thus indicating that a complete separation of the sugars had not been achieved. However, fraction 3 obtained from each parent culture showed a sugar which corresponded in mobility to authentic cellobiose ; also, a similar fraction obtained from organisms $N C I B 8132$ and $N C I B \quad 7029$ showed a sugar corresponding in movement on the paper to cellotriose. These findings were supported by chromatography of these sugars as their $N$-benzylamine derivatives? No attempt was made to identify other sugars present. Examination of the five mutant cultures failed to reveal sugars with mobilities similar to authentic cellobiose or cellotriose in any fraction.

The accumulation of cellobiose and cellotriose in cellulose-producing cultures and the absence of detectable amounts of these sugars in cultures of celluloseless mutants strengthens the hypothesis that these sugars are concerned in the synthesis of bacterial cellulose, although it is not a rigid proof of this. If 